\title{
Association between tirofiban monotherapy and efficacy and safety in acute ischemic stroke
}

Chunrong Tao ${ }^{1 \dagger}$, Yuyou Zhu ${ }^{1 \dagger}$, Chao Zhang ${ }^{1}$, Jianlong Song ${ }^{1}$, Tianlong Liu', Xiaodong Yuan ${ }^{1}$, Wenwu Luo ${ }^{2}$,

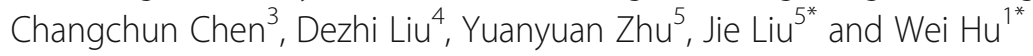

\begin{abstract}
Background: Studies have suggested that glycoprotein IIb/llla antagonists such as tirofiban are beneficial for patients with acute coronary syndromes. However, it is still uncertain about the efficacy and safety of tirofiban in patients with acute ischemic stroke (AIS).

Methods: In this prospective non-randomized study, 255 AlS patients were recruited from 4 comprehensive stroke centers in China between January, 2017 and May, 2018. Among them,169 patients were treated with aspirin plus clopidogrel and 86 patients were treated with tirofiban. The primary functional outcome was the distribution of the 90 days' modified Rankin Scale (mRS). The safety outcomes included the incidence of intracranial hemorrhage (ICH) at discharge and mortality at 3 months.
\end{abstract}

Results: In the propensity score matched cohort, tirofiban alone was noninferior to the dual antiplatelet with regard to the primary outcome (adjusted common odds ratio, $0.97 ; 95 \%$ confidence interval, 0.46 to $2.04 ; P=0.93$ ). Mortality at 90 days was $10 \%$ in the dual antiplatelet group and $8 \%$ in the tirofiban group (adjusted odds ratio 0.75 ; $95 \% \mathrm{Cl} 0.08$ to $7.40, p=0.81$ ). There was no difference of the ICH rate between two groups (adjusted odds ratio $0.44 ; 95 \% \mathrm{Cl} 0.13$ to $1.48, p=0.18$ ). In the inverse probability of treatment weighting-propensity score-adjusted cohort, similar differences were found for functional and safety outcomes.

Conclusions: Our study suggested that tirofiban use appears to be safe as monotherapy in AIS treatment compared with common dual antiplatelet therapy, however, no improvement in functional outcomes was found.

Trial registration: Chinese clinical trial registry, ChiCTR2000034443, 05/07/2020. Retrospectively registered. Keywords: Acute ischemic stroke, Tirofiban, Modified Rankin scale, Mortality

\footnotetext{
* Correspondence: liujie866@126.com; andinghu@ustc.edu.cn

${ }^{\dagger}$ Chunrong Tao and Yuyou Zhu contributed equally to this work.

${ }^{5}$ People's Hospital of LiXin County, BoZhou City 236700, AnHui Province,

China

${ }^{1}$ Stroke Center \& Department of Neurology, Division of Life Sciences and Medicine, the First Affiliated Hospital of USTC, University of Science and Technology of China, Hefei, Anhui, China

Full list of author information is available at the end of the article
}

(C) The Author(s). 2021 Open Access This article is licensed under a Creative Commons Attribution 4.0 International License, which permits use, sharing, adaptation, distribution and reproduction in any medium or format, as long as you give appropriate credit to the original author(s) and the source, provide a link to the Creative Commons licence, and indicate if changes were made. The images or other third party material in this article are included in the article's Creative Commons. licence, unless indicated otherwise in a credit line to the material. If material is not included in the article's Creative Commons licence and your intended use is not permitted by statutory regulation or exceeds the permitted use, you will need to obtain permission directly from the copyright holder. To view a copy of this licence, visit http://creativecommons.org/licenses/by/4.0/ The Creative Commons Public Domain Dedication waiver (http://creativecommons.org/publicdomain/zero/1.0/) applies to the data made available in this article, unless otherwise stated in a credit line to the data. 


\section{Background}

Stroke is currently one of the leading causes of death and disability in China and all over the world [1]. Intravenous thrombolysis (IVT) with alteplase is the preferred therapy for patients with acute ischemic stroke (AIS) [2]. However, the treatment window of IVT is $4.5 \mathrm{~h}$ of symptoms onset, and only $2 \%$ of stroke patients could be beneficial from this treatment regimen [3, 4]. Other treatment options are therefore in demand for the AIS patients who could not receive IVT within the time window.

Antiplatelet therapy is the primary method to prevent and treat AIS [4, 5]. As a highly selective nonpeptide gpIIb/IIIa antagonist, tirofiban has been proven to be beneficial for the treatment of acute coronary syndrome [6]. However, the effect of tirofiban in AIS patients was still uncertain [7, 8]. The effect of platelet aggregation blockade of tirofiban is dose-dependent and can be rapidly reversible in 1.5 to $2.2 \mathrm{~h}$. Therefore, compared with the oral antiplatelet drug clopidogrel and aspirin, intravenous tirofiban infusion is fast-acting and the short half-time makes it possible to swiftly repeal the antiplatelet effect. However, only few studies have evaluated the efficacy of tirofiban and some of them primarily focused on the efficacy of tirofiban in patients receiving IVT or endovascular treatment (EVT) $[9,10]$.

Three studies evaluating the safety and efficacy of tirofiban monotherapy suggested that the functional outcomes of AIS patients could not be improved. Junghans et al. found that the proportion of recovery, stable deficit or slight deterioration were not different $(p=0.18)$ between tirofiban group ( $n=18$, progressive deteriorating AIS) and matched controls ( $n=17$, stable status AIS) [11]. Randomly assigned 150 AIS patients to tirofiban or aspirin, Torgano et al. found that the reduction of $\mathrm{Na}$ tional Institutes of Health Stroke Scale (NIHSS) great than 4 points and the mRS at 3 months were not different between the both groups [12]. Siebler et al. conducted a randomized study with 260 AIS patients, which reported no statistically significant difference of the modified Rankin Scale (mRS) and Barthel Index at 5 months between the tirofiban group and the placebo controlled group [13]. However, mortality at 5 months was significantly lower in tirofiban group (OR, 4.05; 95\% CI, 1.1 to $14.9, p=0.03)$. To date, the effect of tirofiban monotherapy is still controversial in terms of long-term function outcomes and mortality, and no study has yet evaluated the efficacy and safety of tirofiban monotherapy in the East Asian population or using dual antiplatelet as the control group.

Therefore, we present the data of a prospective multicenter trial on the efficacy and safety of tirofiban within the first $24 \mathrm{~h}$ of AIS.

\section{Methods}

\section{Study design}

This was a non-randomized, interventional study, where consecutive patients with AIS treated with tirofiban monotherapy or aspirin plus clopidogrel were enrolled prospectively at 4 stroke centers in China between January 2017 and May 2018. The patients were assigned to either tirofiban or control group in ratio of 1:2. The choice to treatment was determined by the clinical experience of the clinicians and by the patient preference. The intervention group received intravenous continuous infusion of tirofiban while the control group received standard dual antiplatelet treatment (aspirin and clopidogrel).

The study protocol was approved by the medical ethics committee of the first affiliated hospital of USTC. Written informed consent was given by all patients or their relatives before participation.

\section{Eligibility criteria}

The inclusion criteria were as follows: age $\geq 18$ years; on admission within $24 \mathrm{~h}$ after symptom onset. The exclusion criteria were: known thrombocytopenia at presentation or a thrombocyte count of $100 \times 10^{9} / \mathrm{L}$ or lower; had intracranial hemorrhage (ICH); had malignant edema; renal insufficiency (creatinine clearance rate $<30$ $\mathrm{mL} / \mathrm{minutes}$ ); and hepatic dysfunction (serum alanine transaminase $>$ twice the upper limit of the normal value, or serum aspartate transaminase >twice the upper limit of the normal value); pregnant women; subjects disabled before the recent stroke $(m R S>2)$; recent major bleedings, surgery, or trauma.

\section{Medication}

In the tirofiban group, patients received body-weight adjusted intravenous tirofiban infusion at the dose of 0.4 $\mu \mathrm{g} / \mathrm{kg} / \mathrm{min}$ for $30 \mathrm{~min}$ followed by a continuous infusion of $0.1 \mu \mathrm{g} / \mathrm{kg} / \mathrm{min}$ for $48 \mathrm{~h}$. After the infusion of tirofiban, patients received clopidogrel (75 $\mathrm{mg}$ per day) plus aspirin (100 to $200 \mathrm{mg}$ per day). In the antiplatelet group, patients received clopidogrel (75 $\mathrm{mg}$ per day) plus aspirin (100 to $200 \mathrm{mg}$ per day) at the beginning of treatment.

\section{Brain imaging}

After the first cerebral CT examination to exclude primary hemorrhage or findings unrelated to the diagnosis of acute ischemic stroke, a second equally standardized cerebral CT examination was performed 2 to 6 days after the completion of the study medication. Information about the extent of early cerebral ischemia on baseline was measured by the Alberta Stroke Program Early Computed Tomography Score (ASPECTS). 


\section{Data collection and assessment}

Baseline demographic and clinical information for all enrolled patients were recorded, including age, sex, premorbid mRS, admission NIHSS score, stoke etiology, presence of hypertension, diabetes mellitus, hyperlipidemia, atrial fibrillation, smoking history, time from stroke onset to hospital admission and time from hospital admission to the treatment.

Our primary outcome was the scores on the mRS assessed at 90 days (within a window of \pm 14 days). Ninety days after the acute event, functional outcome was assessed by boardcertified vascular neurologists during a routinely scheduled clinical visit or by a study nurse certified in administering the mRS during a standardized telephone interview if the patient was unable to attend. Secondary outcomes included favorable outcome (90-day mRS score 0-3), excellent outcome (90-day mRS score 0-2), 24-h NIHSS shift (defined as baseline NIHSS to 24-h NIHSS) and 7 day or discharge NIHSS shift (defined as baseline NIHSS to 7 day or discharge NIHSS). Safety outcomes included the all-causes mortality at 90 days and the occurrence of cerebral hemorrhage according to the ECASS II (European Collaborative Acute Stroke Study) classification [14]. Imaging criteria were evaluated by a local investigator of each center.

\section{Statistical analyses}

Continuous variables were described as mean and SD, and categorical variables were presented as absolute frequencies. Baseline characteristics were described according to the administered treatment, and the absolute standardized difference (ASD) was used to assess the magnitude of the between-group differences. A standardized difference of $10 \%$ is equivalent to a phi coefficient of 0.05 (negligible correlation), therefore, an ASD of 0.10 or more indicates that covariates are imbalanced between groups [15-17]. We compared the outcomes between the 2 study groups after taking into account the potential confounding factors by using prespecified propensity score methods (PSM) [18].

The effects of the treatment were estimated by using propensity score matching as primary analysis and by using inverse probability weighted regression adjustment (IPWRA) model (probability weights was obtained to

Table 1 Baseline characteristics according to anti-platelet approach in AIS Patients before and after PSM

\begin{tabular}{|c|c|c|c|c|c|c|}
\hline & \multicolumn{3}{|l|}{ Before Matching } & \multicolumn{3}{|l|}{ After Matching } \\
\hline & $\begin{array}{l}\text { Dual antiplatelet } \\
(n=169)\end{array}$ & $\begin{array}{l}\text { Tirofiban } \\
(n=86)\end{array}$ & $\overline{\text { ASD, } \%}$ & $\begin{array}{l}\text { Dual antiplatelet } \\
(n=50)\end{array}$ & $\begin{array}{l}\text { Tirofiban } \\
(n=50)\end{array}$ & ASD, \% \\
\hline Age, $y$, mean (sd) & $72.4(10.1)$ & $72.4(10.3)$ & 0.4 & $70.1(11.2)$ & $70.7(10.9)$ & 5.4 \\
\hline Female, no. (\%) & $72(42.6)$ & $40(46.5)$ & 7.9 & $23(46)$ & $22(44)$ & 4.0 \\
\hline Smoke ever, no. (\%) & $30(17.8)$ & $24(27.9)$ & 24.4 & $8(16)$ & $8(16)$ & 0 \\
\hline Premorbid mRS, mean (sd) & $0.4(0.7)$ & $0.4(0.7)$ & 2.0 & $0.5(0.8)$ & $0.5(0.8)$ & 5.2 \\
\hline \multicolumn{7}{|l|}{ Stroke etiology } \\
\hline Large artery atherosclerosis & $133(78.7)$ & $74(86.0)$ & 22.10 & $42(84)$ & $41(82)$ & 5.6 \\
\hline Cardioembolism & $3(1.8)$ & $2(2.3)$ & & $1(2)$ & $1(2)$ & \\
\hline Other & $33(19.5)$ & $10(11.6)$ & & $7(14)$ & $8(16)$ & \\
\hline ASPECTS, mean (sd) & $7.9(2.4)$ & $8.3(1.8)$ & 22.3 & $8.2(1.9)$ & $7.9(2)$ & 15.2 \\
\hline Admission SBP, mm Hg, mean (sd) & $155.4(28.6)$ & $141.9(23.5)$ & 51.7 & $147(27.9)$ & $143.9(23.3)$ & 12.2 \\
\hline Prior use of IV thrombolysis, no. (\%) & $11(6.5)$ & $7(8.1)$ & 6.3 & $4(8)$ & $5(10)$ & 7.0 \\
\hline Endovascular treatment, no. (\%) & $28(16.6)$ & $13(15.1)$ & 4.0 & $7(14)$ & $8(16)$ & 5.6 \\
\hline NIHSS score prior to treatment, mean (sd) & $8(3.6)$ & $11.7(5.5)$ & 80.0 & $10.1(3.6)$ & $10(5.1)$ & 2.7 \\
\hline \multicolumn{7}{|l|}{ Medical history } \\
\hline Hypertension, no. (\%) & $137(81.1)$ & 18.7 & 18.7 & $38(76)$ & $38(76)$ & 0 \\
\hline Diabetes, no. (\%) & $25(14.8)$ & 10.2 & 10.2 & $7(14)$ & $8(16)$ & 5.6 \\
\hline Hyperlipidemia, no. (\%) & $11(6.5)$ & 14.2 & 14.2 & $2(4)$ & $4(8)$ & 16.9 \\
\hline Atrial fibrillation, no. (\%) & $19(11.2)$ & 4.8 & 4.8 & $4(8)$ & $5(10)$ & 7.0 \\
\hline Coronary disease & $56(33.1)$ & 22.1 & 22.1 & $12(24)$ & $11(22)$ & 4.8 \\
\hline \multicolumn{7}{|l|}{ Workflow, median (IQR), min } \\
\hline onset to admission, h, mean (sd) & $7.5(3.9)$ & 6.1 & 6.1 & $8.1(4.5)$ & $8(3.7)$ & 2.4 \\
\hline onset to treatment, $\mathrm{h}$, mean (sd) & $9.8(4.2)$ & 13.8 & 13.8 & $10.7(5)$ & $10.3(4.7)$ & 6.4 \\
\hline
\end{tabular}

Abbreviations: ASPECTS Alberta Stroke Program Early CT Score, IQR interquartile range, $m R S$ modified Rankin Scale, ASD absolute standardized difference, IV intravenous, NIHSS National Institutes of Health Stroke Scale, SBP systolic blood pressure, EVT endovascular treatment 
calculate the outcome-regression parameters that account for the missing-data problem arising from the fact that each subject is observed in only one of the potential outcomes) as a secondary analysis.

A multivariable probit regression model was used to calculate the propensity score, and using antithrombotic therapy as dependent variable and all the obtained variables related to the outcomes as covariates. Patients in the dual antiplatelet group were matched 1:1 to patients in the tirofiban group based on corresponding PSM, using the nearest neighbor matching algorithm with a caliper width of 0.2 of the propensity score. To evaluate bias reduction after PSM, absolute standardized differences were calculated again after PSM $[19,20]$.

In IPWRA model, between-group comparisons were done with a three-step approach. Firstly, the parameters of the treatment model and the inverse-probability weights were calculated. Secondly, fit weighted regression models of the outcome for each treatment level and obtain the treatment-specific predicted outcomes for each subject with the estimated inverse-probability weights. Thirdly, calculate the means of the treatment-specific predicted outcomes, and the contrasts of these averages provide the estimates of the average treatment effect on the treated [21].

Statistical testing was conducted at the 2-tailed level of 0.05. All analyses were performed using STATA version 14.2 and the significance level was set at 0.05 .

\section{Data availability}

Anonymized data will be shared by request from any qualified investigators.

\section{Results}

Between January 2017 and May 2018, a total of 255 AIS patients were admitted at the participating centers and satisfied the inclusion criteria. Of these, 169 patients were treated with aspirin plus clopidogrel and 86 patients were treated with tirofiban. The primary outcome of the mRS score at 90 days was missing for 18 patients (12 patients in the control group and 6 patients in the intervention group), and data were not imputed.

Fifty matched pairs were found in the primary analysis. Table shows the baseline characteristics according to the 2 study groups before and after PMS. Before matching, smoking history, stroke etiology, ASPECTS score, admission SBP and admission NIHSS showed meaningful differences (ASD > 10\%). ASD reduced significantly after PSM with a maximum ASD of 0 for smoking history, $5.6 \%$ for TOAST classification, $15.2 \%$ for ASPECT score, $12.2 \%$ for admission SBP and $2.7 \%$ for NIHSS score (Table 1).

\section{Efficacy}

In the PSM cohort, the adjusted common odds ratio for the $\mathrm{mRS}$ score at 90 days was 0.97 (95\% confidence interval $[\mathrm{CI}], 0.46$ to 2.04; $P=0.93$ ) (Fig. 1a). Favorable outcome was not different between the control group (32\%) and the intervention group (34\%, matched odds ratio $=1.25,95 \% \mathrm{CI}, 0.38$ to $4.06, p=0.71)$. As shown in Fig. 2a, the improvement of NIHSS after $24 \mathrm{~h}$ showed no significant difference between the two groups $(p=0.64)$ : -1.22 for the dual antiplatelet group and -1.00 for the tirofiban group, respectively. No association was seen

Outcomes
Propensity-score matched cohort
mRS improvement
mRS (0-2)
mRS (0-3)
Death
any ICH
Inverse probability weighted analysis
mRS improvement
mRS (0-2)
mRS (0-3)
Death
any ICH

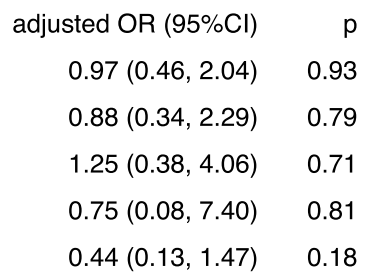

p

.79

.71

0.18

$p$
0.93
0.79
0.71
0.81
0.18

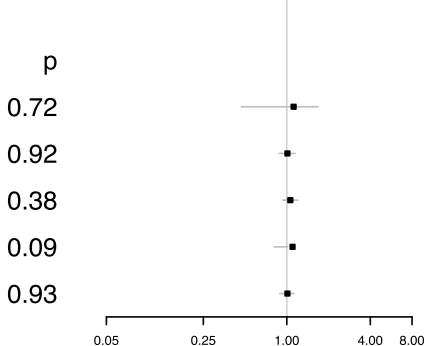

Fig. 1 Comparisons in mRS score, mortality and ICH outcomes according to treatment allocation in AIS patients in propensity-score matched and inverse probability weighted analyses. Abbreviations: $\mathrm{Cl}=$ confidence interval; $\mathrm{OR}=$ odds ratio; $\mathrm{mRS}=$ modified Rankin Scale. All regression analyses were adjusted for the following variables: age, sex, smoking history, baseline Alberta Stroke Program Early CT Score, baseline NIH Stroke Scale score, IV thrombolysis, endovascular treatment, premorbid mRS, TOAST classification, hypertension, diabetes mellitus, hyperlipidemia, atrial fibrillation, coronary disease, onset to admission and onset to treatment 


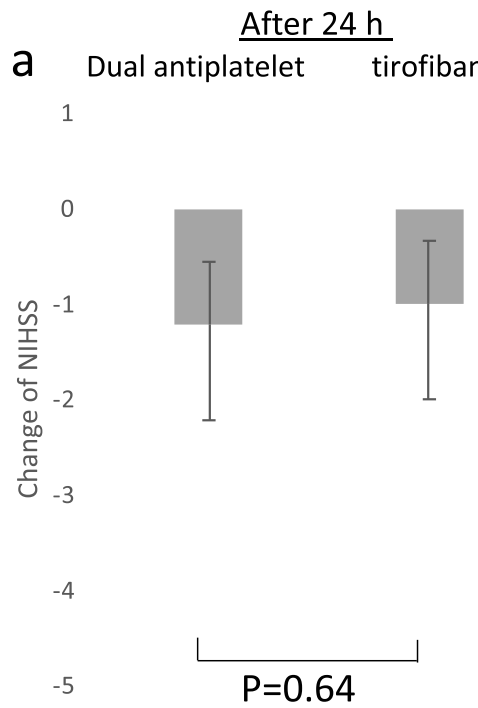

After $24 \mathrm{~h}$

b Dual antiplatelet tirofiban

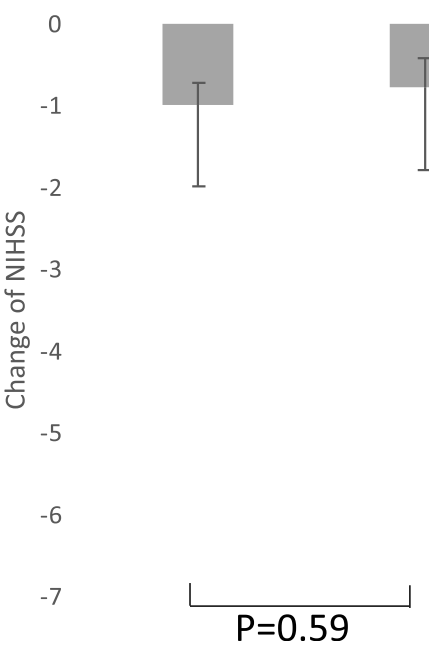

At day 7 or discharge

Dual antiplatelet tirofiban

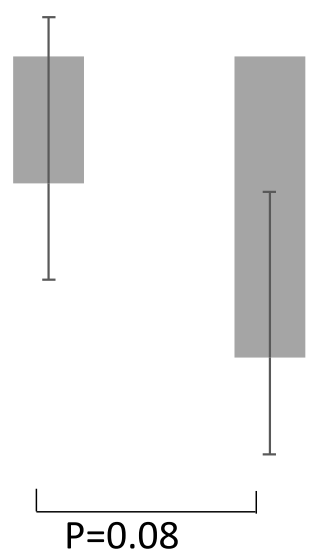

At day 7 or discharge

Dual antiplatelet tirofiban
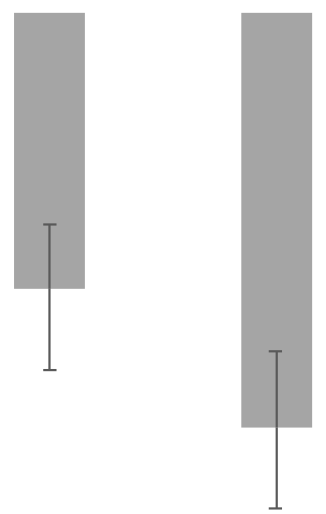

$\mathrm{P}=0.07$

Fig. 2 Association of treatment allocation with NIHSS change at after $24 \mathrm{~h}$ or at day 7 or hospital discharge in (a) propensity-score matched and (b) inverse probability weighted analyses. Abbreviation: NIHSS = National Institutes of Health stroke scale. All regression analyses were adjusted for the following variables: age, sex, smoking history, baseline Alberta Stroke Program Early CT Score, baseline NIH Stroke Scale score, IV thrombolysis, endovascular treatment, premorbid mRS, TOAST classification, hypertension, diabetes mellitus, hyperlipidemia, atrial fibrillation, coronary disease, onset to admission and onset to treatment

between treatment allocation and improvement in NIHSS at 7 days as well $(p=0.08)$. In IPWRA model, similar results were found for efficacy outcomes (Figs. 1 and 2b).

\section{Safety}

In the PSM cohort (Fig. 1a), mortality at 90 days was $8.0 \%$ in the control group and $10.0 \%$ in the intervention group (adjusted odds ratio 0.75 . $95 \%$ CI 0.08 to 7.40 , $p=0.81)$. There was no difference $(p=0.18)$ of the percentages of patients with $\mathrm{ICH}$ in the dual antiplatelet group $(24 \%$ [12 patients] $)$ and in the tirofiban monotherapy group (8\% [16 patients]). The sensitivity analysis with IPWRA model provided similar results (Fig. 1b).

\section{Discussion}

Our study was designed to test the efficacy and safety of tirofiban in patients with AIS. We found that tirofiban use was not associated with mRS score at 3-month, and the risk of $\mathrm{ICH}$ as well as the mortality were not increased compared with the standard dual antiplatelet care. 
The optimal antithrombotic approach during AIS is still uncertain. Patients with the administration of tirofiban showed no improvement in functional outcome as measured by mRS at 3 months, which was in agreement with the previous studies evaluating the efficacy and safety of tirofiban monotherapy in non-selected AIS population [11-13, 22]. In AIS patients receiving EVT, studies have also demonstrated that the use of tirofiban could not increase the proportion of patients with $\mathrm{mRS}$ $0-2$ at 3 months $[9,10,23-30]$. Studies failed to reach a consensus about whether the efficacy of tirofiban was superior in patients receiving IVT with alteplase [31, 32].

Our study also found that the reduction of NIHSS at $24 \mathrm{~h}$ and at 7 days or discharge were similar between two groups. Torgano et al. found that the reduction of NIHSS $\geq 4$ was seen in $56 \%$ of cases in both tirofiban group and aspirin group [12]. Including 82 patients, Li et al. found that NIHSS score at 7 days was significantly lower with alteplase plus tirofiban as compared to alteplase alone $(p=0.002)$ [5]. The association between tirofiban monotherapy and the early neurological functional change could not be determined yet, and the future prospective cohort study is needed to clarify this association.

There was no significant difference in the incidence of $\mathrm{ICH}$ between the two groups. Several studies have also demonstrated that the combination of tirofiban and other standard cares did not increase the risk of ICH $[13,31,33]$. It can be inferred that the administration of tirofiban instead of dual antiplatelet is relatively safe in AIS patients who had fulfilled the inclusion criteria.

Our study found that the mortality was similar between the control group and the tirofiban group after the 3 months' follow-up. In line with our results, one recent meta-analysis demonstrated that the mortality at 3 months did not increase after the administration of tirofiban (OR, 0.80; 95\% CI; 0.64-1.02; $p=0.07$ ) [22]. Wu et.al performed a multicenter retrospective cohort study including 187 Chinese patients found that tirofiban use was associated with an insignificant but lower mortality at 3 months after IV thrombolysis (OR, 0.77; 95\% CI, 0.19-2.27; $P=0.875$ ) [32].

There are several limitations of this study. First, despite that we used propensity score analysis to minimize the difference in baseline characteristics, our results could be confounded by variables that were not included in the propensity model. Second, when evaluating the long-term functional outcome, adjunct therapies administered to the patients in the first 3 months following stroke were not controlled for and may have influenced the outcome. Third, relatively small sample size prevented us from performing further stratification analysis in the PMS cohort.

\section{Conclusion}

Through a more specifically and reversibly binding to platelet GP IIb/IIIa receptors, and a shorter half-life of 2 $\mathrm{h}$, tirofiban use was associated with a similar rate of hemorrhagic complications whether it is administered alone or following IVT or EVT. An acceptable efficacy and safety profile allowed for alternative or adjuvant treatment to current management of early AIS. For AIS patients at high risk of refractoriness or progression, the use of tirofiban have been providing a new perspective for the validated treatment. Its inhibition effect on ongoing platelet aggregation and thrombosis, rather than absolute thrombolytic effect suggests that tirofiban may be promising in future AIS treatment.

To the best of our knowledge, this is the first study evaluating the association between tirofiban monotherapy within the $24 \mathrm{~h}$ after symptom onset and prognosis in AIS patients in the East Asian population. Our results indicate that tirofiban could not improve functional outcomes compared to the standard dual antiplatelet care in AIS patients, but the low $\mathrm{ICH}$ rate and mortality suggested that it is safe when administered early as monotherapy. Further prospective cohort studies with large sample size are required to provide robust evidence.

\begin{abstract}
Abbreviations
AIS: Acute ischemic stroke; mRS: Modified Rankin Scale; ICH: Intracranial hemorrhage; NIHSS: National Institutes of Health Stroke Scale; ASPE CTS: Alberta Stroke Program Early Computed Tomography Score; ASD: Absolute standardized difference; PSM: Propensity score methods; IPWRA: Inverse probability weighted regression adjustment; OR: Odds ratio
\end{abstract}

\section{Acknowledgements}

We thank all patients, caregivers, health care professionals, students and institutions who have contributed and collaborated in this study.

\section{Authors' contributions}

Conception and design: $W H, J L, C T$ and $Y Z 1$. Analysis and interpretation: $C T$, $Y Z 1$, and $W H$. Collecting the data: $C Z, J S, T L, X Y, W L, C C, D L$ and $Y Z 2$. Drafting the manuscript and checking for important intellectual content: $C T$ and WH. All authors critically read and revised the manuscript, and approved its submission for publication.

\section{Funding}

This study was funded by the Key Research and Development Projects of Anhui Province (No: 201904a07020086). The study was designed, conducted, analyzed and interpreted by the investigators independent of sponsors.

\section{Availability of data and materials}

The datasets used and/or analysed during the current study available from the corresponding authors $(\mathrm{WH}$ and $\mathrm{J}$ ) on reasonable request.

\section{Declarations}

\section{Ethics approval and consent to participate}

The study is conducted in accordance with the Declaration of Helsinki. Potential participants or their relatives receive detailed written information on the study procedures and all participants provide written informed consent. Ethics approval of the study was obtained from the medical ethics committee of the first affiliated hospital of USTC.

Consent for publication

Not applicable. 


\section{Competing interests}

The authors declare that they have no competing interests.

\section{Author details}

${ }^{1}$ Stroke Center \& Department of Neurology, Division of Life Sciences and Medicine, the First Affiliated Hospital of USTC, University of Science and Technology of China, Hefei, Anhui, China. ${ }^{2}$ Department of Pathology, The First Affiliated Hospital of Anhui Medical University, Hefei, Anhui, China. ${ }^{3}$ Department of Neurology, The Second People's Hospital of Anhui Province, Hefei, Anhui, China. ${ }^{4}$ Department of Neurology, Shuguang Hospital Affiliated to Shanghai University of TCM, 528 Zhang-Heng Road,Pu-Dong New Area, Shanghai 201203, China. ${ }^{5}$ People's Hospital of LiXin County, BoZhou City 236700, AnHui Province, China.

\section{Received: 16 November 2020 Accepted: 4 June 2021} Published online: 24 June 2021

\section{References}

1. Zhou M, Wang H, Zhu J, et al. Cause-specific mortality for 240 causes in China during 1990-2013: a systematic subnational analysis for the Globa Burden of Disease Study 2013. Lancet (London, England). 2016;387(10015): 251-72.

2. Powers WJ, Rabinstein AA, Ackerson T, Adeoye OM, Bambakidis NC, Becker K, et al. Guidelines for the early Management of Patients with Acute Ischemic Stroke: 2019 update to the 2018 guidelines for the early Management of Acute Ischemic Stroke: A guideline for healthcare professionals from the American Heart Association/American Stroke Association. Stroke. 2019;50(12):e344-418. https://doi.org/10.1161/STR. 0000000000000211

3. Wahlgren N, Ahmed N, Dávalos A, et al. Thrombolysis with alteplase for acute ischaemic stroke in the Safe Implementation of Thrombolysis in Stroke-Monitoring Study (SITS-MOST): an observational study. Lancet (London, England). 2007;369(9558):275-82.

4. Kernan WN, Ovbiagele B, Black HR, et al. Guidelines for the prevention of stroke in patients with stroke and transient ischemic attack: a guideline for healthcare professionals from the American Heart Association/American Stroke Association. Stroke. 2014:45(7):2160-2236.

5. Li W, Lin L, Zhang M, et al. Safety and preliminary efficacy of early tirofiban treatment after alteplase in acute ischemic stroke patients. Stroke. 2016; 47(10):2649-51.

6. Rognoni A, Lupi A, Secco GG, et al. Update on glycoprotein Ilb/lla: role in primary coronary intervention. Cardiovasc Hematol Agents Med Chem. 2011;9(2):106-12.

7. Ciccone A, Motto C, Abraha I, Cozzolino F, Santilli I. Glycoprotein IIb-Illa inhibitors for acute ischaemic stroke. Cochrane Database Syst Rev. 2014(3): CD005208.

8. Cheng Z, Geng X, Gao J, et al. Intravenous administration of standard dose tirofiban after mechanical arterial recanalization is safe and relatively effective in acute ischemic stroke. Aging Dis. 2019;10(5):1049-57.

9. Kellert L, Hametner C, Rohde S, et al. Endovascular stroke therapy: tirofiban is associated with risk of fatal intracerebral hemorrhage and poor outcome. 2013:44(5):1453-5. https://doi.org/10.1161/STROKEAHA.111.000502.

10. Pan $X$, Zheng $D$, Zheng $Y$, et al. Safety and efficacy of tirofiban combined with endovascular treatment in acute ischaemic stroke. 2019;26(8):1105-10. https://doi.org/10.1007/s00234-019-02282-1.

11. Junghans U, Seitz RJ, Aulich A, Freund HJ, Siebler M. Bleeding risk of tirofiban, a nonpeptide GPIlb/Illa platelet receptor antagonist in progressive stroke: an open pilot study. Cerebrovasc Dis (Basel, Switzerland). 2001;12(4): 308-12.

12. Torgano G, Zecca B, Monzani V, et al. Effect of intravenous tirofiban and aspirin in reducing short-term and long-term neurologic deficit in patients with ischemic stroke: a double-blind randomized trial. Cerebrovasc Dis (Basel, Switzerland). 2010:29(3):275-81.

13. Siebler M, Hennerici MG, Schneider $D$, et al. Safety of Tirofiban in acute ischemic stroke: the SaTIS trial. Stroke. 2011;42(9):2388-92.

14. Hacke W, Kaste M, Fieschi $C$, et al. Randomised double-blind placebocontrolled trial of thrombolytic therapy with intravenous alteplase in acute ischaemic stroke (ECASS II). Second European-Australasian Acute Stroke Study Investigators. Lancet (London, England). 1998;352(9136):1245-51.

15. Normand ST, Landrum MB, Guadagnoli $\mathrm{E}$, et al. Validating recommendations for coronary angiography following acute myocardial infarction in the elderly: a matched analysis using propensity scores. J Clin Epidemiol. 2001; 54(4):387-98. https://doi.org/10.1016/S0895-4356(00)00321-8.

16. Austin P. Using the standardized difference to compare the prevalence of a binary variable between two groups in observational research. Commun Stat Simul Comput. 2009;38(6):1228-34. https://doi.org/10.1080/0361091 0902859574

17. Austin P. Balance diagnostics for comparing the distribution of baseline covariates between treatment groups in propensity-score matched samples. Stat Med. 2009;28(25):3083-107. https://doi.org/10.1002/sim.3697.

18. Austin P. An introduction to propensity score methods for reducing the effects of confounding in observational studies. Multivar Behav Res. 2011; 46(3):399-424. https://doi.org/10.1080/00273171.2011.568786.

19. Austin P. A comparison of 12 algorithms for matching on the propensity score. Stat Med. 2014;33(6):1057-69. https://doi.org/10.1002/sim.6004.

20. Austin PC. Optimal caliper widths for propensity-score matching when estimating differences in means and differences in proportions in observational studies. Pharm Stat. 2011;10(2):150-61. https://doi.org/10.1 002/pst.433

21. Cattaneo MD. Efficient semiparametric estimation of multi-valued treatment effects under ignorability. J Econ. 2010;155(2):138-54. https://doi.org/10.101 6/j.jeconom.2009.09.023.

22. Gong J, Shang J, Yu H, et al. Tirofiban for acute ischemic stroke: systematic review and meta-analysis. Eur J Clin Pharmacol. 2020;76(4):475-81.

23. Yang M, Huo X, Gao F, Wang A, Ma N, Shi H, et al. Low-dose rescue tirofiban in mechanical thrombectomy for acute cerebral large-artery occlusion. Eur J Neurol. 2020;27(6):1056-61. https://doi.org/10.1111/ene.141 70

24. Zhang Y, Zhang QQ, Fu C, Wang L, Zhang GQ, Cao PW, et al. Clinical efficacy of tirofiban combined with a solitaire stent in treating acute ischemic stroke. Braz J Med Biol Res. 2019;52(10):e8396. https://doi.org/10.1 590/1414-431×20198396.

25. Yi HJ, Sung JH, Lee DH. Safety and efficacy of intra-arterial Tirofiban injection during mechanical Thrombectomy for large artery occlusion. Curr Neurovasc Res. 2019;16(5):416-24. https://doi.org/10.2174/15672026166661 91023154956.

26. Sun C, Li X, Zhao Z, Chen X, Huang C, Li X, et al. Safety and efficacy of Tirofiban combined with mechanical Thrombectomy depend on ischemic stroke etiology. Front Neurol. 2019;10:1100. https://doi.org/10.3389/fneur.201 9.01100 .

27. Quan T, Hou H, Xue W, Yu G, Ma H, Sun J, et al. Endovascular treatment of acute intracranial vertebrobasilar artery occlusion: a multicenter retrospective observational study. Neuroradiology. 2019;61(12):1477-84. https://doi.org/10.1007/s00234-019-02282-1.

28. Yu T, Lin Y, Jin A, Zhang P, Zhou X, Fang M, et al. Safety and efficiency of low dose intra-arterial Tirofiban in mechanical Thrombectomy during acute ischemic stroke. Curr Neurovasc Res. 2018;15(2):145-50. https://doi.org/10.21 74/1567202615666180605104931.

29. Zhao W, Che R, Shang S, Wu C, Li C, Wu L, et al. Low-dose Tirofiban improves functional outcome in acute ischemic stroke patients treated with endovascular Thrombectomy. Stroke. 2017;48(12):3289-94. https://doi.org/1 0.1161/STROKEAHA.117.019193.

30. Luo $Y$, Yang $Y$, Xie Y, Yuan Z, Li X, Li J. Therapeutic effect of pre-operative tirofiban on patients with acute ischemic stroke with mechanical thrombectomy within 6-24 hours. Interv Neuroradiol. 2019;25(6):705-9. https://doi.org/10.1177/1591019919851167.

31. Liu J, Shi Q, Sun Y, et al. Efficacy of Tirofiban administered at different time points after intravenous thrombolytic therapy with Alteplase in patients with acute ischemic stroke. J Stroke Cerebrovascr Dis. 2019;28(4):1126-32.

32. Wu C, Sun C, Wang L, et al. Low-dose Tirofiban treatment improves neurological deterioration outcome after intravenous thrombolysis. Stroke. 2019;50(12):3481-7.

33. Ma Q, Ma Y, Wang X, et al. Intracoronary compared with intravenous bolus tirofiban on the microvascular obstruction in patients with STEMI undergoing PCl: a cardiac MR study. Int J Cardiovasc Imaging. 2020;36(6): $1121-32$.

\section{Publisher's Note}

Springer Nature remains neutral with regard to jurisdictional claims in published maps and institutional affiliations. 